\title{
Intravenous drug use - an independent predictor for HCV genotypes 3 and 4 infection among HIV/HCV co-infected patients
}

Dubravka Salemovic ${ }^{1}$, Ivana Pesic-Pavlovic ${ }^{2}$, Djordje Jevtovic ${ }^{1,3}$, Ksenija Bojovic ${ }^{1}$, Jovan Ranin ${ }^{1,3}$, Branko Brmbolic ${ }^{1,3}$, Maja Stanojevic ${ }^{4}$

\author{
${ }^{1}$ Infectious and Tropical Diseases University Hospital, Clinical Center Serbia, HIV/AIDS \\ Unit, Belgrade, Serbia \\ ${ }^{2}$ Virology Department, Clinical Center Serbia, NRL HIV/AIDS, Belgrade, Serbia \\ ${ }^{3}$ University of Belgrade Faculty of Medicine, Belgrade, Serbia \\ ${ }^{4}$ Institute of Microbiology and Immunology, NRL HIV/AIDS, University of Belgrade \\ School of Medicine, Belgrade, Serbia
}

Submitted: 19 January 2015

Accepted: 2 July 2015

Arch Med Sci 2017; 13, 3: 652-658

DOI: https://doi.org/10.5114/aoms.2017.66022

Copyright $\odot 2017$ Termedia \& Banach

\section{Abstract}

Introduction: About one quarter of human immunodeficiency virus (HIV) infected persons in Serbia have also been found to be hepatitis C virus (HCV) co-infected. In the general population, HCV genotype 1 has been shown to be the most prevalent one. Here, we present the first study on the distribution of HCV genotypes among HIV/HCV co-infected patients in Serbia, in relation to epidemiological and clinical features.

Material and methods: The study included HIV/HCV co-infected and a group of HCV mono-infected patients in the period 1998-2012, with collection of epidemiological, clinical, and behavioral data using a standardized questionnaire. The HCV genotyping to the level of pure genotype was performed by reverse hybridization.

Results: Intravenous drug use (IDU) was found to be significantly more prevalent among the co-infected patients $(p<0.01)$. HCV genotype 1 was detected in $87 \%$ of patients with mono-infection, compared to $46.3 \%$ of patients with co-infection $(p<0.01)$; genotypes 3 and 4 were significantly more common among co-infected patients $(6 \%$ and $5 \%$, vs. $27 \%$ and $25 \%$, respectively). Multivariate logistic regression confirmed IDU, infection with non-1 HCV genotype and HCV viral load over 5 log to be predictors of HIV co-infection. Conclusions: The HCV genotypes 3 and 4 were found to be significantly more prevalent among HIV/HCV co-infected patients in Serbia, compared to HCV mono-infected patients, but also more prevalent compared to the European HIV/HCV co-infected cohort. History of IDU represents an independent predictor of HCV genotypes 3 and 4 infection, with important implications for treatment.

Key words: intravenous drug use, human immunodeficiency virus/ hepatitis C virus co-infection, HCV genotypes, Serbia.

\section{Introduction}

With an estimated number of people seropositive for hepatitis $C$ virus (HCV) worldwide approaching 200 million, HCV and its associated liver disease (cirrhosis, end stage liver disease, hepatocellular carcinoma)

\author{
Corresponding author: \\ Prof. Maja Stanojevic PhD \\ Institute of Microbiology \\ and Immunology \\ NRL HIV/AIDS \\ University of Belgrade \\ School of Medicine \\ 11000 Belgrade, Serbia \\ Phone: +38 1645104296 \\ E-mail: mstanojevic@med. \\ bg.ac.rs
}


are among the leading causes of morbidity and mortality globally [1]. Human immunodeficiency virus (HIV) and HCV share the same transmission routes - they are both transmitted via parenteral, sexual, and vertical exposure, although with different transmission efficiencies. The reported prevalence of HIV/HCV coinfection varies significantly among studies, ranging from $4 \%$ up to $90 \%$, depending on the geographical region and studied population [2]. Both HIV and HCV are characterized by substantial genetic variability, resulting in the existence of multiple viral genotypes, subtypes and variants. According to the most recent nomenclature proposal, HCV is classified into 7 genotypes, further subdivided into epidemiologically diverse subtypes [3]. Genotype distribution varies widely, both geographically and related to different transmission categories, and has a major impact on HCV infection therapy [4].

Serbia is a South East European country, situated on the Balkan Peninsula, with a population of about 7 million. According to World Bank data, Serbia is ranked as an upper middle income country; however, for decades already it has been passing through a dramatic period of economic transition as well as political and social changes, potentially facilitating spread of infectious diseases associated with intravenous drug use (IDU) and sexual transmission. The HIV epidemic in Serbia has been present for almost three decades and was first introduced among IDUs [5]. According to the latest epidemiological data, among newly diagnosed HIV infections in 2014, the proportion of IDUs has decreased to around $4 \%$, while the infection rate among men who have sex with men (MSM) has increased, becoming the most prevalent transmission route among newly diagnosed infections in Serbia, with around 60\% (http:// www.batut.org.rs/index.php?content=1095). The HIV infection cases are not evenly distributed throughout the country, with the majority of cases reported in the capital Belgrade metropolitan area.

In Serbia, about one quarter of HIV-infected persons (24.3\%) have also been found to be HCV co-infected [6]. Existing data on the prevalence of diverse HCV genotypes in the general population in Serbia depict genotype 1 as the most prevalent one [7]. So far, no data are available regarding HCV genotype distribution among HIV/HCV co-infected patients. In order to gain insight into the epidemiology, molecular characteristics and risk factors for HCV infection in the HIV-infected population in Serbia, we performed a study examining the distribution of prevalent HCV genotypes among HIV/ $\mathrm{HCV}$ co-infected patients in Serbia and comparing it to the one in HCV mono-infected patients, in relation to various epidemiological and clinical features.

\section{Material and methods}

The study prospectively included consenting HIV/HCV co-infected and a group of HCV monoinfected patients followed at the Infectious and Tropical Diseases University Hospital in Belgrade, according to the following inclusion criteria: ELISA determined and western blot confirmed seropositive status for HCV mono-infection or HIV/HCV co-infection and adult age. The study included patients in the period 1998-2012. Epidemiological, clinical, and behavioral data were collected using a standardized questionnaire. Transmission risk was defined as IDU, sexual contact, history of transfusion of blood/blood products, vertical and other. The study was conducted under the approval of the Ethical Committee of the Clinical Center of Serbia.

HIV infection was diagnosed using the Enzygnost HIV Integral II test (Siemens Healthcare Diagnostics, Marburg, Germany) and confirmed by the NEW LAV-BLOT II western blot test (BIORAD, Marnes La Coquette, France). HIV viral load was measured using the Cobas TaqMan HIV Test (Roche Molecular Systems, Branchburg, NJ, USA). HCV infection was diagnosed by antibody detection using Monolisa Anti-HCV PLUS Version 2 (BIO-RAD, Marnes La Coquette, France).

For patients followed in the period 2010-2012, HCV RNA and genotyping was performed using commercial tests. HCV viral load was measured by the COBAS TaqMan HCV Test (Roche Molecular Systems, Branchburg, NJ, USA). A viral load above $100 \mathrm{IU} / \mathrm{ml}$ was needed for further HCV genotyping, which was performed by reverse hybridization, using the LINEAR ARRAY Hepatitis C Virus Genotyping Test (Roche Molecular Systems, Branchburg, NJ, USA).

For HCV/HIV co-infected patients, HIV disease was classified in stages A-C according to the 1993 Centers for Disease Control case definition criteria [8]. Aspiration liver biopsy was performed in order to assess severity of fibrosis and necroinflammatory activity, using the Ishak modification of the hepatic activity index. In some patients, liver biopsy was not performed due to liver failure, presence of hemangiomas or other reasons (e.g. patients' reluctance to undergo the procedure). The diagnosis of cirrhosis was established using histological or a combination of clinical, endoscopic and laboratory findings.

\section{Statistical analysis}

Obtained results were processed using standard statistical analysis. All analyses were performed using an electronic database organized in the SPSS (version 11.5) statistical package. Categorical data were compared using the $\chi^{2}$ test and 
Fisher's exact test. Plasma HCV viral load means were compared by one-way ANOVA. Univariate and multivariate logistic regression was used to study the association between HCV/HIV co-infection and different epidemiological and clinical variables (age, HCV genotype, HCV viral load above $5 \log _{10} \mathrm{IU} / \mathrm{ml}$, risk of acquiring HIV/HCV infection) and also to assess variables associated with HCV genotypes 3 and 4 infection (age below 40, i.v. drug use and HIV co-infection). The level of statistical significance was 0.05 .

\section{Results}

The study included $214 \mathrm{HIV} / \mathrm{HCV}$ co-infected and $193 \mathrm{HCV}$ mono-infected patients who fulfilled the inclusion criteria. Co-infected patients presented in different stages of HIV disease: 29/214 patients were CDC A (13.6\%), 72/214 patients were in stage B (33.6\%), whereas $113 / 214$ patients presented with CDC C (52.8\%). General characteristics of studied patients are presented in Table I. Patients were mostly male (58.0\%), which did not differ between the two subgroups, while those co-infected were significantly younger. The prevalence of transmission risk was found to be substantially different in the group of HIV/ HCV co-infected patients, compared to the mono- infected ones. The majority of co-infected patients $(151 / 214,70.5 \%)$ were IDUs, compared to $37 / 193$ (19.1\%) HCV mono-infected patients $(p<0.01)$; sexual transmission was reported by $2.5 \%$ of mono-infected, compared to $22.8 \%$ of co-infected patients $(p<0.01)$ (Figure 1$)$.

For 229 patients followed in the period 20102012, HCV RNA and genotyping was performed. HCV genotype distribution was found to be significantly different between the two groups: genotype 1 was detected in $87 \%$ of patient with monoinfection, compared to $46.3 \%$ of patients with coinfection $(p<0.01)$; non-1 genotypes, namely genotypes 3 and 4 , were significantly more common among co-infected patients ( $6 \%$ and $5 \%$, vs. $27 \%$ and $25 \%$, respectively) (Figure 2). Non-1 HCV genotypes were more commonly found among IDUs compared to other transmission risks, regardless of the HIV status ( $p=0.03)$. HCV plasma viral load was significantly higher in co-infected patients $\left(6.0 \pm 1.2\right.$, vs. $\left.4.4 \pm 1.7 \log _{10} I U / l, p=0.01\right)$ (Figure 3). However, viral load did not differ significantly across different genotypes, regardless of HIV co-infection. In addition, end stage liver disease (liver cirrhosis) was not related to certain genotypes, and surprisingly was even more prevalent among mono-infected subjects. Cirrhotic patients from the mono-infected subgroup were signifi-

Table I. General clinical and demographic characteristics of patients

\begin{tabular}{|c|c|c|c|c|}
\hline Parameter & Total & HCV mono-infection & HIV/HCV co-infection & $P$-value \\
\hline Patients & 407 & 193 & 214 & \\
\hline Age, mean $\pm S D$ & & $41.7 \pm 13.9$ & $37.5 \pm 7.8$ & 0.01 \\
\hline Male, $n(\%)$ & $236(58 \%)$ & $115(59.6 \%)$ & $121(56.5 \%)$ & 0.3 \\
\hline Female, $n(\%)$ & $171(42 \%)$ & 78 (40.4\%) & 93 (43.5\%) & 0.3 \\
\hline \multicolumn{5}{|l|}{ CDC stage, $n(\%)$ : } \\
\hline A & & & $29(13.6 \%)$ & \\
\hline B & & & $72(33.6 \%)$ & \\
\hline c & & & $113(52.8 \%)$ & \\
\hline $\begin{array}{l}\text { HCV hepatitis* } \\
\text { Liver cirrhosis }\end{array}$ & 94 & 56 & 38 & 0.002 \\
\hline \multicolumn{5}{|l|}{ Transmission risk: } \\
\hline IDU (\%) & $188(46.2 \%)$ & 37 (19.2\%) & $151(70.5 \%)$ & $<0.01$ \\
\hline Sexual & $54(13.3 \%)$ & $5(2.6 \%)$ & $49(22.9 \%)$ & $<0.01$ \\
\hline Parenteral & $130(31.9 \%)$ & $117(60.6 \%)$ & $13(6.1 \%)$ & $<0.001$ \\
\hline MTC & $1(0.2 \%)$ & 0 & $1(0.5 \%)$ & \\
\hline Unknown & $34(8.4 \%)$ & 34 (17.6\%) & 0 & \\
\hline $\begin{array}{l}\mathrm{HCV} \text { RNA }[\mathrm{IU} / \mathrm{ml}] \log _{10} \\
\pm \mathrm{SD}\end{array}$ & & $4.6 \pm 1.6$ & $6.0 \pm 1.2$ & $<0.01$ \\
\hline
\end{tabular}

* Liver cirrhosis data were available for 182 mono-infected and 213 co-infected patients. IDU - intravenous drug use, parenteral - history of transfusion of blood/blood products, MTC - mother-to-child transmission. 


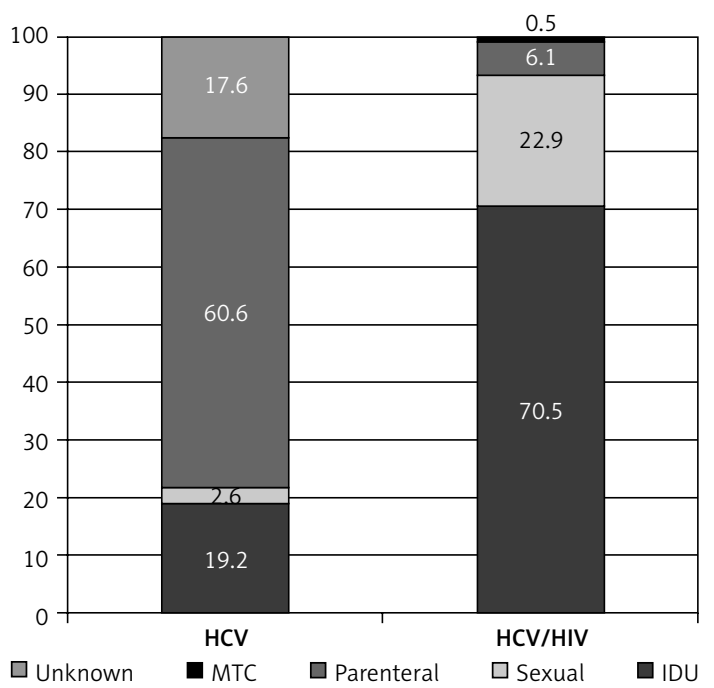

Figure 1. Transmission risk distribution, in percentages, among HCV mono-infected and HIV/HCV co-infected patients

MTC - mother-to-child transmission, parenteral - history of transfusion of blood/blood products, IDU - intravenous drug use.

cantly older than those co-infected with liver cirrhosis ( $48.7 \pm 12.3$ vs. $38.5 \pm 7.2, p=0.01$ ). Logistic regression analyses revealed that in the whole cohort of HCV infected patients, certain HCV genotypes were not associated with a higher incidence of liver cirrhosis, while age above 40 was an independent predictor of ESLD $(\mathrm{OR}=2.7,95 \% \mathrm{Cl}$ : 1.7-4.5, $p=0.01$ ).

Univariate logistic regression identified IDU, age under 40, infection with non-1 HCV genotype and HCV viral load over 5 log to be associated with HIV co-infection, whereas multivariate logistic regression confirmed IDU, infection with non-1 $\mathrm{HCV}$ genotype and HCV viral load over 5 log to be predictors of HIV co-infection. Univariate logistic regression showed that age below 40, i.v. drug use and HIV co-infection were associated with genotypes 3 and 4, while multivariate logistic regression revealed i.v. drug use to be an independent predictor of acquiring genotype 3 or 4 .

\section{Discussion}

Here, we present the first comprehensive study of the HCV genotype distribution among HIV/HCV co-infected patients in Serbia.

HCV-associated liver disease has been proven as a major cause of morbidity and mortality worldwide, affecting an estimated $2-3 \%$ of the world's population, and particularly HIV-infected persons $[9,10]$. Available data indicate that HCV seroprevalence varies considerably worldwide, with country-specific prevalence ranging from $<1 \%$ to $>10 \%$ [10]. In Europe, high diversity in prevalence of HCV infection in the general population has been described, ranging from $0.4 \%$ to $22 \%$, with higher

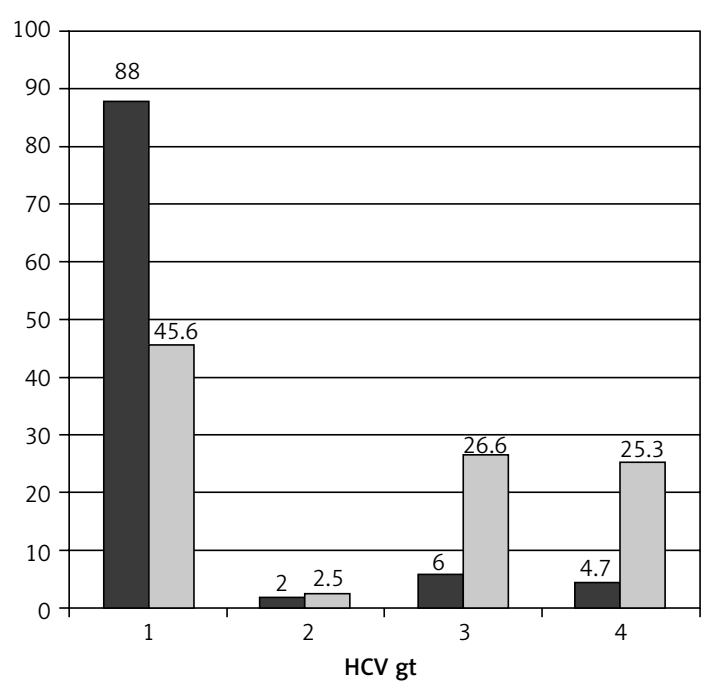

$\square$ HCV mono-infection $\quad \square$ HIV/HCV co-infection

Figure 2. HCV genotype distribution, in percentages, among HCV mono-infected and HIV/HCV co-infected patients; HCV gt 1-4 - hepatitis $C$ virus pure genotypes $1-4$

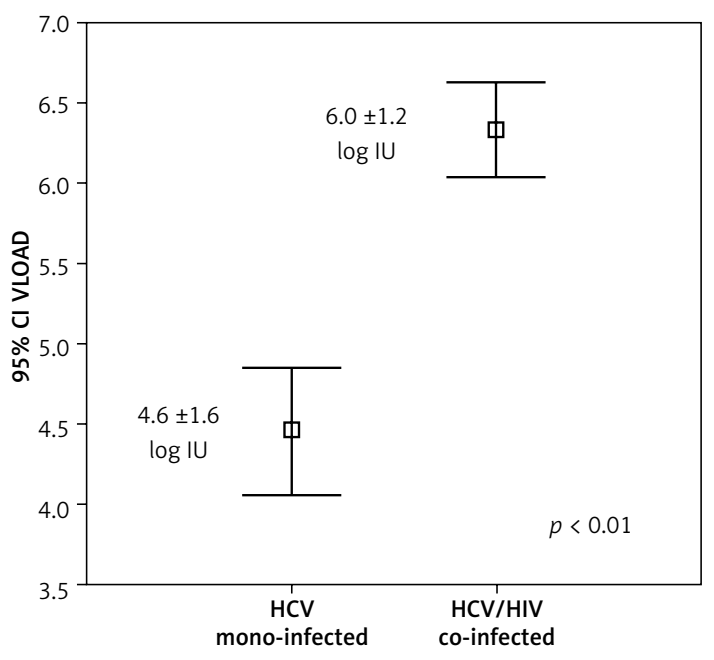

Figure 3. Average HCV plasma viral load among HCV mono-infected and HIV/HCV co-infected patients

rates in southern and central-eastern countries [11]. Low prevalence $(\leq 0.5 \%)$ has been described in Belgium, Germany, Netherlands, Sweden, and the UK, intermediate prevalence $(0.5-2 \%)$ in Bulgaria, France, Hungary, and Romania, whereas high prevalence $(\geq 2 \%$ ) has been found in parts of Italy [11]. Still, data on general population prevalence are lacking or incomplete for many countries and regions of the world. According to the Serbian Public Health Institute, the registered annual incidence of chronic HCV infection in Serbia was in the range of 5.86-7.32 per 100000 , for the period 2008-2012 [12]. However, the actual prevalence of HCV infection and the true burden of disease are not known - a recent global survey has esti- 
mated regional seroprevalence for Central Europe to be $2.4 \%(2.0-2.8)$, which can be classified as "moderate" [1]. Regarding HIV infection, Serbia ranks among countries with low prevalence (less that $0.1 \%$ ), with the incidence rate of HIV-1 infection of 17 per million in 2012 [13].

The HIV/HCV co-infection is known to induce important liver-related morbidity and mortality. Even in the highly active antiretroviral era, HIV co-infection remains independently associated with advanced liver fibrosis and cirrhosis in patients with chronic HCV infection [14]. In our cohort of HCV- infected patients, the prevalence of HCV-related end-stage liver disease (ESLD, namely, liver cirrhosis) was even higher among the HCV mono-infected ones, probably because these patients were significantly older than those concurrently-infected with HIV. However, HCV mono-infected patients with liver cirrhosis were significantly older than those with liver cirrhosis and HCV/HIV co-infection, suggesting more rapid development of cirrhosis among co-infected patients. The HIV coinfection has been shown to adversely affect the natural history of HCV disease, increasing the risk of decompensated liver disease or histological cirrhosis in co-infected patients, compared to patients with HCV infection alone [15]. Accelerated progression of hepatic fibrosis has been linked to enhanced HCV replication, presumably due to HIV-related immunosuppression [16]. Higher HCV viral load has been described in our cohort, as one of the potential contributors to enhanced liver fibrosis. Molecular mechanisms of accelerated hepatic fibrosis among HIV/HCV co-infected individuals have been attributed to direct viral effects, immune/cytokine dysregulation, altered levels of matrix metalloproteinases and fibrosis biomarkers, increased oxidative stress and hepatocyte apoptosis, HIV-associated gut depletion of CD4 cells, and microbial translocation [17]. Recently, HCV-related cirrhosis was found to be associated with the level of immune activation and elevated serum concentrations of monocyte-derived markers [18].

A study concerning a Belgrade cohort of Serbian patients evaluated and treated between January 1998 and December 2007 at the HIV/AIDS Centre of the Infectious and Tropical Diseases University Hospital in Belgrade identified HCV co-infection in $24.3 \%$ [6]. It has been shown that the prevalence of HIV/HCV co-infection is directly influenced by transmission risk factors of the population under study. Parenteral transmission through intravenous drug use, history of multiple transfusions or blood product exposures have been described as the most important risk factors for HIV/HCV co-infection [19]. In HIV-positive patients with a history of IDU, the rate of HCV infection is reported to be $82 \%$ to $93 \%$. Conversely, the rate of co-infection among HIV-infected patients with a sexual risk factor is much lower, less than $10 \%$, related to the relatively low HCV transmission rate via sexual exposure [19]. In our study, the prevalence of transmission risk was found to be substantially different between the HCV mono-infected and HIV co-infected patients. Accidental parenteral exposure to blood, along with contaminated blood and/or blood product transfusions were the most prevalent ways of transmission among HCV mono-infected patients, while in the subgroup of $\mathrm{HCV} / \mathrm{HIV}$ co-infected subjects parenteral exposure to contaminated needles, among IDUs, and sexual acquisition of the infection were more common. In our study, IDUs were significantly younger than all other HCV-infected patients, which mostly contributed to the mean age of HIV co-infected patients being significantly lower than among monoinfected patients.

Besides the shared transmission routes, both HIV and HCV are rapidly evolving RNA viruses, presenting substantial intra- and inter-host genetic variability. Six well-established HCV genotypes described so far and the newly described genotype 7 are found, with differing prevalence worldwide [20]. Several HCV subtypes of genotypes 1, 2 and 3 are globally distributed, and hence termed epidemic, whereas others, the endemic ones, are much more restricted to particular geographic regions [21]. Genotypes $1 \mathrm{a}$ and $1 \mathrm{~b}$ are the most prevalent in western countries, with genotype $1 \mathrm{~b}$ being the most common in Europe and genotype $1 \mathrm{a}$ in the United States [20]. Genotype 2 and its subtypes predominate in western Africa. Genotype $3 a$ is the second most common genotype in Europe after genotype 1 . Genotype 4 is found in Central Africa, the Mediterranean region and the Middle East [22-25]. Subtype 5 a seems to have originated in South Africa but is also present in Europe, whereas genotype 6 is common in Southeast Asia [22-24, 26, 27]. In Serbia, previous studies have found HCV genotype 1 to be the most prevalent one in the general population, accounting for $57.9 \%$ of chronic infections, followed by subtypes 3, 4 and 2, found in $23.2 \%, 6.7 \%$ and $3.7 \%$ of patients, respectively [7].

The HCV genotype distribution has been found to reflect the route of transmission: genotype $1 \mathrm{~b}$ has been found associated with post-transfusion HCV infections, whereas HCV subtypes $1 \mathrm{a}$ and 3 have been repeatedly associated with IDU, in different regions of the world [28, 29]. Several studies have described higher prevalence of HCV genotype 6 among HCV patients with a history of IDU, specifically in some regions of China [30, 31]. The HCV genotypes have not been found to differ with regard to disease severity, but they still affect treatment duration and response, even with new direct-acting antivirals (DAA) [25]. 
In our study, the HCV genotype distribution found among HIV/HCV co-infected patients in Serbia was substantially different compared to HCV mono-infected patients, with much higher prevalence of non-1 genotypes. In particular, genotypes 3 and 4 were found to be significantly more prevalent in co-infected patients. In Serbia, the duration of the HIV epidemic is similar to the one in Western European countries, with the first cases registered in 1985. The HIV epidemic in Serbia was first recognized among IDUs, and this transmission route was the most prevalent one over the coming years. By the end of the 1990s, intravenous drug use was still the most prevalent risk factor among cumulative HIV/AIDS cases, around $48.9 \%$, followed by sexual transmission, 33\% [32]. However, according to the latest epidemiological data, men who have sex with men (MSM) have become the most prevalent transmission route among newly diagnosed infections in Serbia [33, 34]. The difference we found in genotype 3 distribution reflects higher prevalence of IDUs among the co-infected. Similar to other regions of the world $[28,29]$, in Serbia, HCV subtype $3 a$ has been found to be associated with IDU transmission, whereas a study comparing HCV genotype distribution in chronically HCV infected patients with and without a history of IDU found significantly higher prevalence of genotypes 3 and 2 and lower prevalence of genotypes 1 and 4 in the former group [7, 31]. Notably, we found a quarter of co-infected patients (25.3\%) to harbor HCV subtype 4, compared to less than $5 \%$ in the mono-infected group. Genotype 4 is known to have originated in Central Africa, but is increasingly present in Southern Europe and in the Mediterranean region (France, Spain, Greece and Italy) $[21,28,35]$. It has been described in co-infected patients previously, although in a lower percentage than in our study (ranging from $0.8 \%$ to $20 \%)[36,37]$. Recently, an increase in genotype 4 prevalence has been described in England [38], mostly associated with countries with endemic presence of this subtype. Detection of HCV genotype 4 infection in the European cohort of HIV/HCV co-infected patients in the last decade has been attributed to the wider use of HCV therapy and also linked to immigrants from endemic countries [39]. Neither of these reasons plays an important role in the Serbian HIV/HCV co-infected cohort, whereas we found that high local prevalence of HCV genotype 4 is related to i.v. drug use as a risk for HCV infection. The transmission dynamics of HCV have been shown to vary by subtype and to be highly influenced by a combination of age, risk exposure and underlying social network [40]. Hence social factors, rather than biological features of different genotypes, may play a key role in shaping the rate and pattern of HCV spread.
Similar to other commercially available HCV genotyping methods, the one used in our study, reverse hybridization, is used to characterize the tested HCV genome at the level of pure genotype. Reverse hybridization is considered very reliable, with the reported percentage of genotyping failure using this method being less than $5 \%[40,41]$. However, we obtained no insight into HCV subgenotype distribution in the studied population (e.g. $4 a$ vs. $4 b$ ), which could be considered a possible limitation to our study.

In conclusion, HCV genotype distribution among HIV/HCV co-infected patients in Serbia is substantially different compared to HCV mono-infected patients, with much higher prevalence of genotypes 3 and 4 . The proportion of HCV genotype 4 infection is also considerably higher compared to the European HIV/HCV co-infected cohort. We found that this difference is linked to higher prevalence of IDU among the co-infected in Serbia. However, the association of particular HCV genotypes with specific routes of transmission and with HIV co-infection warrants further investigation. Detailed understanding of HCV genotypes' prevalence and distribution is essential for an effective targeted treatment and prevention approach.

\section{Acknowledgments}

This work was partially funded by the Ministry of Education and Science, Republic of Serbia (grant no. 175024). We thank Dr Sonja Zerjav for valuable help in study design and technical performance.

\section{Conflict of interest}

The authors declare no conflict of interest.

\section{References}

1. Mohd Hanafiah K, Groeger J, Flaxman AD, Wiersma ST. Global epidemiology of hepatitis $C$ virus infection: new estimates of age-specific antibody to HCV seroprevalence. Hepatology 2013; 57: 1333-42.

2. Rockstroh JK, Spengler U. HIV and hepatitis C virus co-infection. Lancet Infect Dis 2004; 4: 437-44.

3. Smith DB, Bukh J, Kuiken C, et al. Expanded classification of hepatitis $C$ virus into 7 genotypes and 67 subtypes, updated criteria and assignment web resource. Hepatology 2014; 59: 318-27.

4. Shepard CW, Finelli L, Alter MJ. Global epidemiology of hepatitis C virus infection. Lancet Infect Dis 2005; 5: 558-67.

5. Zerjav S. Fridman V, Suvakovic V, et al.: Epidemiologija AIDS-a u Beogradu [Serbian]. Srp Arh Celok Lek 1987; 115: 715-23.

6. Jevtović DJ, Salemović D, Ranin J, Dulović O, llić D, Brmbolić $B$. The prognosis of highly active antiretroviral therapy (HAART) treated HIV infected patients in Serbia, related to the time of treatment initiation. J Clin Virol 2010; 47: 131-5. 
7. Svirtlih N, Delic D, Simonovic J, Jevtovic D, et al. Hepatitis $C$ virus genotypes in Serbia and Montenegro: the prevalence and clinical significance. World J Gastroenterol 2007; 13: 355-60.

8. 1993 revised classification system for HIV infection and expanded surveillance case definition for AIDS among adolescents and adults. MMWR Recomm Rep 1992; 41: $1-19$.

9. Weber R, Sabin CA, Friis-Møller N, et al. Liver-related deaths in persons infected with the human immunodeficiency virus: the D:A:D study. Arch Intern Med 2006; 166: $1632-41$

10. Averhoff FM, Glass N, Holtzman D. Global burden of hepatitis $C$ : considerations for healthcare providers in the United States. Clin Infect Dis 2012; 55: S10-5.

11. Cornberg M, Razavi HA, Alberti A, et al. A systematic review of hepatitis Cvirus epidemiology in Europe, Canada and Israel. Liver Int 2011; 31 Suppl. 2: 30-60.

12. European Centre for Disease Prevention and Control. Hepatitis B and C in the EU neighbourhood: prevalence, burden of disease and screening policies. ECDC; Stockholm 2010.

13. Obrenovic J, Grgic B, Loncarevic G, et al. Godišnji izveštaj o zaraznim bolestima u 2012. godini na teritoriji Republike Srbije. Institut za Javno Zdravlje Srbije: Centar zaprevencij uikontrolubolesti; 2013 [Annual report on communicable diseases in 2012 in the Republic of Serbia. Institute for Public Health of Serbia: Centre for prevention and control of diseases; 2013].

14. European Centre for Disease Prevention and Control/ WHO Regional Office for Europe. HIV/AIDS surveillance in Europe 2012. Stockholm: European Centre for Disease Prevention and Control; 2013.

15. Graham CS, Baden LR, Yu E, et al. Influence of human immunodeficiency virus infection on the course of hepatitis $C$ virus infection: a meta-analysis. Clin Infect Dis 2001; 33: 562-9.

16. Martinez-Sierra C, Arizcorreta A, Dıaz F, et al. Progression of chronic hepatitis $C$ to liver fibrosis and cirrhosis in patients coinfected with hepatitis $C$ virus and human immunodeficiency virus. Clin Infect Dis 2003; 36: 491-8.

17. Mastroianni CM, Lichtner M, Mascia C, Zuccalà P, Vullo V. Molecular mechanisms of liver fibrosis in HIV/HCV coinfection. Int J Mol Sci 2014; 15: 9184-208.

18. Márquez M, Romero-Cores P, Montes-Oca M, et al. Immune activation response in chronic HIV-infected patients: influence of hepatitis C virus coinfection. PLoS One 2015; 10: e0119568.

19. Thein HH, Yi Q, Dore GJ, Krahn MD. Natural history of hepatitis $C$ virus infection in HIV-infected individuals and the impact of HIV in the era of highly active antiretroviral therapy: a meta-analysis. AIDS 2008; 22: 1979-91.

20. Rotman Y, Jake Liang T. Co-infection with hepatitis C virus and human immunodeficiency virus: virological, immunological, and clinical outcomes. J Virol 2009; 83: 7366-74.

21. Manos MM, Shvachko VA, Murphy RC, Arduino JM, Shire NJ. Distribution of hepatitis $C$ virus genotypes in a diverse US integrated health care population. J Med Virol 2012; 84: 1744-50.

22. Jackowiak P, Kuls K, Budzko L, Mania A, Figlerowicz M, Figlerowicz M. Phylogeny and molecular evolution of the hepatitis C virus. Infect Genet Evol 2014; 21 : 67-82.

23. Markov PV, Pepin J, Frost E, Deslandes S, Labbe AC, Pybus OG. Phylogeography and molecular epidemiology of hepatitis C virus genotype 2 in Africa. J Gen Virol 2009; 90: 2086-96.
24. Njouom R, Caron M, Besson G, et al. Phylogeography, risk factors and genetic history of hepatitis $C$ virus in Gabon, central Africa. PLoS One 2012; 7: e42002.

25. Messina JP, Humphreys I, Flaxman A, et al. Global distribution and prevalence of hepatitis $C$ virus genotypes. Hepatology 2015; 61: 77-87.

26. Pybus OG, Barnes E, Taggart R, et al. Genetic history of hepatitis C virus in East Asia. J Virol 2009; 83: 1071-82.

27. Verbeeck J, Maes P, Lemey P, et al. Investigating the origin and spread of hepatitis $C$ virus genotype $5 \mathrm{a}$. J Virol 2006; 80: 4220-6.

28. Cornberg M. A systematic review of hepatitis C virus epidemiology in Europe, Canada and Israel. Liver Int 2011; 31 Suppl. 2: 30-60.

29. Treso B, Takacs M, Dencs A, Dudas M, Par A, Rusvai E. Molecular epidemiology of hepatitis $C$ virus genotypes and subtypes among injecting drug users in Hungary. Euro Surveill 2013; 18 pii: 20639.

30. Tian D, Li L, Liu Y, Li H, Xu X, Li J. Different HCV genotype distributions of HIV-infected individuals in Henan and Guangxi, China. PLoS One 2012; 7: e50343.

31. Zhang Z, Yao Y, Wu W, et al. Hepatitis C virus genotype diversity among intravenous drug users in Yunnan Province, Southwestern China. PLoS One 2013; 8: e82598.

32. Bojovic K, Simonovic J, Katanic N, et al. The comparison of chronic hepatitis $C$ treatment outcome between intravenous drug users and non-intravenous drug users. Biomed Pharmacother 2013; 67: 517-20.

33. Stanojevic M, Papa A, Papadimitriou E, et al. HIV-1 subtypes in Yugoslavia. AIDS Res Hum Retrovir 2002; 18 . 519-26.

34. Siljic $M$, Salemovic D, Jevtovic D, et al. Molecular typing of the local HIV-1 epidemic in Serbia. Infect Genet Evol 2013; 19: 378-85.

35. Iles JC, Raghwani J, Harrison GL, et al. Phylogeography and epidemic history of hepatitis C virus genotype 4 in Africa. Virology 2014; 464-465C: 233-43.

36. Cifuentes C, Mancebo-Hernández M, Pérez-Navarro E, et al. Prevalence and genotype distribution changes in hepatitis $C$ virus co-infection among human immunodeficiency virus-infected patients [Spanish]. Enferm Infecc Microbiol Clin 2015; 33: 110-2.

37. Petruzziello A, Coppola N, Loquercio G, et al. Distribution pattern of hepatitis $C$ virus genotypes and correlation with viral load and risk factors in chronic positive patients. Intervirology 2014; 57: 311-8.

38. Ngui SL, Brant L, Markov PV, et al. Hepatitis C virus genotype 4 in England: diversity and demographic associations. J Med Virol 2015; 87: 417-23.

39. Medrano J, Resino S, Vispo E, et al. Hepatitis C virus ( $\mathrm{HCV}$ ) treatment uptake and changes in the prevalence of HCV genotypes in HIV/HCV-co-infected patients. J Viral Hepat 2011; 18: 325-30.

40. Romano CM, de Carvalho-Mello IMVG, Jamal LF, et al. Social networks shape the transmission dynamics of hepatitis C virus. PLoS One 2010; 5: e11170.

41. González V, Gomes-Fernandes M, Bascuñana E, et al. Accuracy of a commercially available assay for HCV genotyping and subtyping in the clinical practice. J Clin Virol 2013; 58: 593-7. 